\title{
Right Ventricular Dysfunction After Thrombolysis in Patients with Right Ventricular Infarction
}

\author{
Anna Vittoria M attioli, MD, PhD , FESC, M atteo Fini, M D, and Giorgio M attioli, M D, \\ Modena, I taly
}

\begin{abstract}
Background: Right ventricular (RV) infarction is frequently associated with highest risk of death and major complications. Doppler echocardiography can be useful in the diagnosis of $\mathrm{RV}$ involvement. The goal of this study was to evaluate Doppler echocardiography features associated with RV involvement and a poor prognosis.

Methods Two-dimensional Doppler echocardiography was performed before and after thrombolysis in 108 consecutive patients with an RV infarction. The bedside examination was performed before and 2 to 3 hours after thrombolytic therapy, and repeated after 1 and 7 days. All patients underwent coronary angiography after 20 days, and the perfusion of the coronary-related artery ( $>$ thrombolysis in myocardial infarction [TIMI] 3 grade) was evaluated.
\end{abstract}

\section{INTRODUCTION}

Right ventricular (RV) infarction complicates up to half of inferior left ventricular (LV) infarctions. ${ }^{1}$ It is associated with a high risk of in-hospital death and major complications. 2,3 The management and prognosis of RV infarction differs substantially from LV infarction. ${ }^{4}$ The depressed RV function induces RV failure and low cardiac output.5,6 Right ventricular infarction results from the occlusion of the right coronary artery proximal to the marginal branches. ${ }^{7}$ The effect of reperfusion in RV infarction is somewhat controversial. Some authors have reported the recovery of RV function only after successful reperfusion, 8,9 whereas other authors observed improvement even in the absence of reperfusion. ${ }^{10,11}$

From the D epartment of Cardiology, U niversity of M odena and Reggio Emilia, I taly.

Reprint requests: Anna Vittoria M attioli, MD, Department of Cardiology, U niversity of Modena and Reggio Emilia, Via del pozzo, 71, 41100 M odena, I taly (E-mail: mattioli.annavittoria@unimo.it).

Copyright $\odot 2000$ by the American Society of Echocardiography. 0894-7317/ $2000 \$ 12.00+0 \quad$ 27/ 1/ 105580

doi:10.1067/ mje.2000.105580
R esults Patients were divided into 2 groups according to the recovery of global and regional RV function after thrombolytic therapy. In the group of patients who showed a normalization or improvement of RV wall motion (as assessed by RV wall motion score index), we found a TIMI grade III perfusion in $78 \%$ of patients. The analysis of interatrial septal motion and interventricular septal motion showed a normalization in all reperfused patients. Major complication and deaths were more frequent in patients with echocardiographic findings of $\mathrm{RV}$ dysfunction persisting after thrombolytic therapy.

Condusion: In patients with RV infarction treated with thrombolysis, persistent RV dysfunction is associated with a higher risk for the development of major cardiac complications and death. (J Am Soc Echocardiogr 2000;13:655-60.)

The goal of our study was to evaluate the effect of thrombolytic therapy and of reperfusion on RV dysfunction evaluated by Doppler echocardiography.

\section{METHODS}

Patients

Included in this prospective study were 108 consecutive patients with RV infarction who were admitted to the intensive coronary care unit of our hospital from January 1994 to January 1998. The initial diagnosis of RV infarction was based on typical chest pain lasting more than 30 minutes and values greater than 2 times normal in aspartate transamine $(30 \mathrm{U} / \mathrm{I})$ and creatine phosphokinase (170 U/I) activity within 24 hours after admission. Electrocardiographic changes of ST-segment elevation of $>0.1 \mathrm{mV}$ and/or $\mathrm{Q}$ waves in V4-V6R leads were also required.

\section{Study Protocol}

In all patients, a standard 12-lead electrocardiogram and right precordial leads (V3-V6R) were recorded within 10 
Table 1 Clinical characteristics of subjects

\begin{tabular}{lc}
\hline \multicolumn{1}{c}{$\begin{array}{c}\text { Clinical } \\
\text { characteristics }\end{array}$} & $\begin{array}{c}\text { Right ventricular } \\
\text { infarction }\end{array}$ \\
\hline N umber of patients & 108 \\
M ean age (y) & $65 \pm 9$ \\
M en/ women & $71 / 37$ \\
H eart rate (bpm) & $66 \pm 12$ \\
Systolic blood pressure (mm H g) & $104 \pm 19$ \\
Previous myocardial infarction & 3 \\
H ypertension & 20 \\
Diabetes & 29 \\
\hline
\end{tabular}

hours from the onset of symptoms. ST-segment deviations were assessed 0.04 seconds after the J point in all 16 leads. An RV infarction was diagnosed if there were clinical, electrocardiographic, hemodynamic, and radiologic criteria. The hemodynamic alterations that were considered highly indicative of an RV infarction included right atrial pressure that was significantly elevated and exceeded the pulmonary-capillary wedge pressure. ${ }^{6}$ Right atrial pressure was measured by catheter in 60 patients and was calculated from echocardiography in all patients.

\section{Coronary Angiography}

The coronary angiography was considered indicative of an RV infarction if there was an occlusion of the right coronary artery proximal to the acute marginal branches. ${ }^{12}$ The severity of stenosis and the extent of flow were determined and graded according to the thrombolysis in myocardial infarction (TIMI) classification. ${ }^{13}$ Successful reperfusion was defined as a residual stenosis less than $50 \%$ and restoration of TIMI grade 3 flow in the main right coronary artery, its left ventricular branches, and all major RV branches.

\section{Doppler Echocardiography}

On admission, an echocardiogram was obtained for all patients before and 2 to 3 hours after thrombolytic therapy was started. Echocardiograms were repeated after 1 day and 1 month. Echocardiographic and Doppler examinations were performed with a Hew lett-Packard 1500 (Andover, Mass) echocardiographic system with a $2.5-\mathrm{MHz}$ transducer. The following parameters were recorded.

- Right and left ventricular end-diastolic diameters were recorded in the supine position with the same Mmode cross section in the parasternal long-axis view. Normal values were defined as less than $25 \mathrm{~mm}$ for the RV diameter, greater than $37 \mathrm{~mm}$ (maximal value 54 $\mathrm{mm}$ ) for the LV diameter and an RV diameter of less than half the LV diameter. 14,15

- Right ventricular function was assessed with the ejection fraction and the wall motion score. The RV free wall was divided into 3 segments, and the motion of
Table 2 I maging and D oppler echocardiography findings in subjects before thrombolytic therapy

\begin{tabular}{lrrl}
\hline \multicolumn{1}{c}{ Echocardiographic data } & Group A & Group B & P \\
\hline RV end-diastolic diameter $(\mathrm{cm})$ & $2.9 \pm 3.4$ & $3.0 \pm 3.6$ & $\mathrm{NS}$ \\
LV end-diastolic diameter $(\mathrm{cm})$ & $4.1 \pm 3.2$ & $4.1 \pm 2.8$ & $\mathrm{NS}$ \\
RV wall motion score & $3.0 \pm 0.7$ & $3.2 \pm 0.7$ & $\mathrm{NS}$ \\
M ean RAP & $16.8 \pm 2.5$ & $19.2 \pm 4.1$ & $\mathrm{NS}$ \\
TR flow velocity (m/ s & $2.9 \pm 0.3$ & $3.0 \pm 0.7$ & $\mathrm{NS}$ \\
\hline
\end{tabular}

RV, Right ventricular; N S, not significant; LV, left ventricular; TR , tricuspid regurgitation; RAP, right atrial pressure.

each segment was scored on a scale of 1 to $4(1$, normal; 2 , hypokinetic; 3 , akinetic; 4 dyskinetic). ${ }^{16}$

- Interventricular septal motion was analyzed from the parasternal long-axis and apical 4-chamber views and was considered abnormal if there was paradoxical wall motion of the septum.

- Interatrial septal motion was analyzed from the subcostal and apical 4-chamber views and was considered abnormal if a bowing toward the left atrium was recorded. 17

- From the hepatic vein flow velocity, the following parameters were measured: peak velocity and timevelocity integral of the systolic, diastolic, and atrial reversal waves. The duration of the atrial reversal wave was measured from the beginning to the end of the atrial reversal wave. The inspiratory change was measured from the 2-dimensional subcostal view, from which a percent collapse index was obtained.The inspiratory change, commonly referred to as a sniff test, was used frequently to estimate right atrial pressure. ${ }^{18}$

- Peak velocity of tricuspid regurgitant flow was recorded from parasternal short-axis and apical 4chamber views. ${ }^{19}$

\section{Prognostic Evaluation}

All patients were followed for 1 year. Complications occurring during the in-hospital and follow-up periods were determined for all patients. The major in-hospital complications evaluated were hypotension (systolic blood pressure $<100 \mathrm{~mm} \mathrm{Hg}$ ), cardiogenic shock (systolic blood pressure $<90 \mathrm{~mm} \mathrm{Hg}$ for 30 minutes), second- and thirddegree atrioventricular block, and death. During follow-up, patients were evaluated every month by phone contact and every 2 months by a clinical visit.

\section{Statistical Analysis}

Data are expressed as mean $\pm S D$. Comparisons were made with use of the chi-square test for categorical variables and a 2-tailed student $t$ test for continuous variables. Analysis of variance $w$ as used to compare serial echocardiographic data. A probability $(\mathrm{P})$ value of $<.05$ was considered significant. 


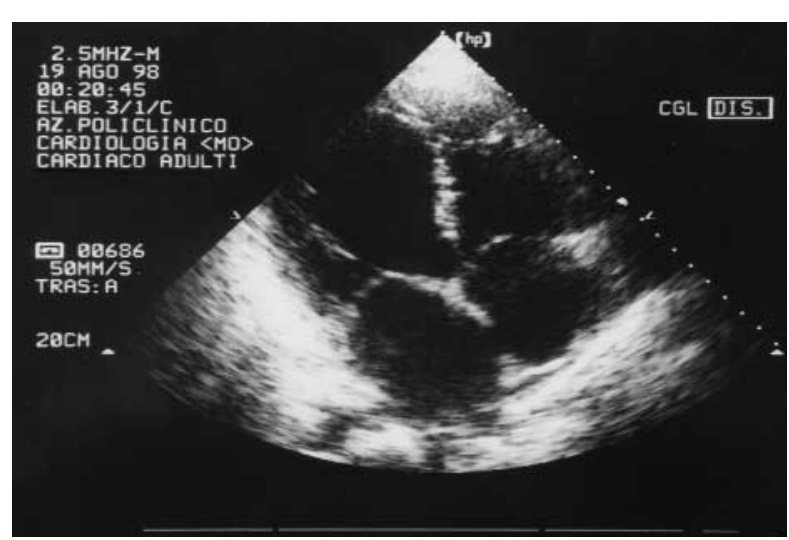

Figure 1 Apical 4-chamber view of a patient with a right ventricular infarction. The right side of the heart is dilated; tricuspid regurgitation was moderate.

\section{RESULTS}

Clinical data of the patients are show $n$ in Table 1.The patients arrived at the hospital a mean of $1.6 \pm 3.5$ hours after the onset of symptoms.

\section{Echocardiographic Features Before Thrombolysis}

Before thrombolysis, all patients had an increase in the RV end-diastolic diameter (Figure 1). Left ventricular end-diastolic diameter was within normal range in all patients except 3 who had a previous myocardial anterior infarction (Table 2). In all patients, analysis of the right regional wall motion showed severe RV dysfunction, with a mean score of $3.2 \pm 0.7$ with paradoxical septal motion. An inverted interatrial septal convexity was observed in all patients (Figure 2).

Nighty-four patients (87\%) had a tricuspid regurgitation detectable by Doppler. In 70 of these patients, flow velocity was increased, compatible with mild to moderate elevation of RV systolic pressure (regurgitant jet $>2.5 \mathrm{~m} / \mathrm{s}$ ). The maximal pressure gradient was $65.8 \pm 27.2$, but most patients had an increased mean right atrial pressure (Table 2 ).

\section{Echocardiographic Variables After Thrombolysis}

Marked changes in echocardiographic variables were seen after thrombolytic therapy in a group of 83 patients (group A).The RV diameter decreased significantly and RV function recovered promptly. As RV function recovered, LV function showed a concomitant improvement (Figure 3). Interventricular septal motion normalized in all patients of Group A. The shape and convexity of the interatrial septum

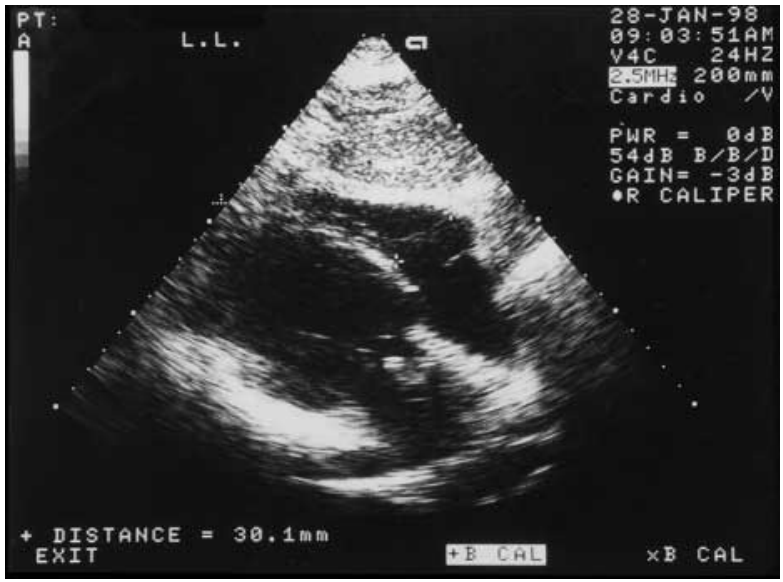

Figure 2 Subcostal 4-chamber view of a patient with right ventricular infarction after 1 month. The right ventricle is slightly dilated; the right ventricular ejection fraction and the right ventricular score are improved compared with the acute phase.

normalized in all patients of Group A, suggesting a decrease in mean right atrial pressure that was confirmed by calculations (from $16.8 \pm 2.5$ to 8.3 $\pm 3.4 ; \mathrm{P}<.01$ ). After 1 month, RV dimension and RV wall motion were normal in $98 \%$ of patients in group A. On the contrary, a group of 25 patients showed a persistence of echocardiographic signs of RV dysfunction after thrombolytic therapy (group B).These patients showed a persistent, severe RV dysfunction at 24 hours (RV size from $30 \pm 3.6 \mathrm{~cm}$ to $29 \pm 3.3$ $\mathrm{cm} ; \mathrm{P}=$ not significant, and RV wall-motion score from $3.2+0.7$ to $2.9+0.4$ ) (Figure 3). The tricuspid regurgitant flow persisted after thrombolytic therapy in all patients, and mean right atrial pressure remained elevated.After 1 month, $78 \%$ of patients of group $B$ had a recovery of RV function with a reduction of RV size and an increase in wall motion score.

\section{Angiographic Findings}

Coronary angiography was performed in all patients after $24 \pm 5$ days from the onset of acute myocardial infarction (range 7 to 35 days). Single-vessel disease was documented in 67 patients, 2-vessel disease in 37 , and 3-vessel disease in 4 . The right coronary artery was involved in all cases. Reperfusion of the right main coronary artery and its LV branches was observed in 80 patients in group $A$ and 5 patients in group $B(P<.01)$.

\section{Prognostic Impact of Right Ventricular Infarction}

The prevalence of major complications and the death rates during the in-hospital phase of the study 
Group A

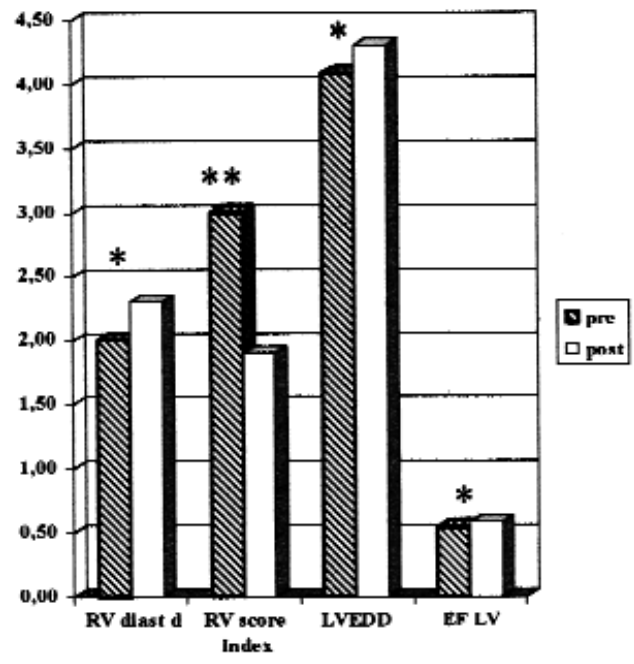

Group B

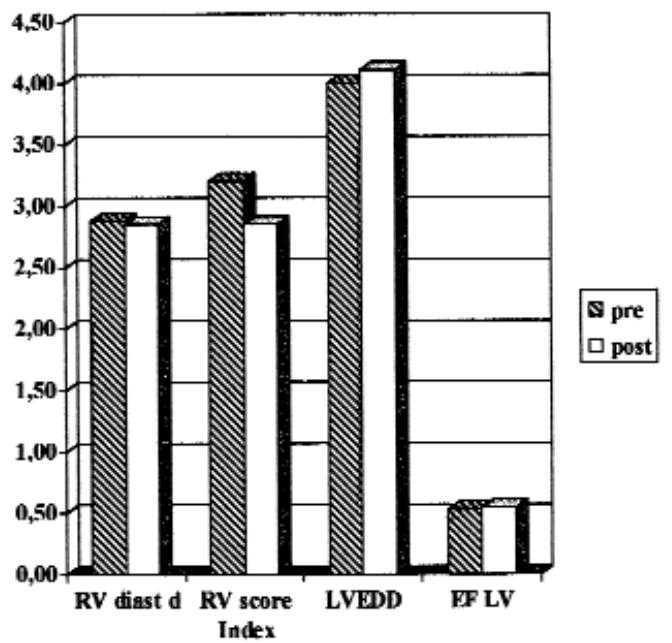

Figure 3 Bar graph showing different parameters before and after thrombolysis in the 2 groups of patients. Group A, Patients who showed a normalization or improvement of RV wall motion (as assessed by RV wall motion score index); group $B$, patients with echocardiographic findings of RV dysfunction persisting after thrombolytic therapy; RV, right ventricular; LVEDD, left ventricular end-diastolic diameter; EF LV, left ventricular ejection fraction.

and after discharge from the hospital were as follows. Hypotension occurred in 32 (29\%) of 108 patients with RV infarction. The incidence of secondand third-degree atrioventricular block was 58 (54\%) out of 108 patients.

Adverse events were rare in the patients from group A.The overall in-hospital mortality rate of the 108 patients with RV myocardial infarction was $12 \%$. Of the 13 patients who died, 9 were from group $B$ and had occlusion of the right coronary artery $(P<.01)$. Seventy-eight $(94 \%)$ patients of group A survived and were discharged from the hospital. At 1 month, all were alive; at 6 months, 68 patients were alive. The mortality (9 [36\%] of 25 patients] and incidence of second- or third-degree AV block (18 [72\%] of 25 patients] were higher in patients of group B. Of the 14 patients of group B who were discharged, 1 died within 1 month, and 3 more died before 6 months.

\section{DISCUSSION}

We report a significant early reversal of RV dilatation and dysfunction in $76.8 \%$ of patients with RV infarction treated with thrombolysis who were successfully reperfused.

Many recent reports stress that the occlusion of the right coronary artery proximal to the major right branches results in RV dysfunction. 12,13,20 The majority of these studies focused their attention on the reperfusion of the LV branches of the right coronary artery, ignoring the affect on RV branches. In a recent report, Bowers et al ${ }^{16}$ suggested that complete reperfusion led to a striking immediate improvement of RV function followed by a complete recovery. In a previous article, we evaluated the effect of thrombolysis on right atrial and ventricular pressures with the use of Doppler echocardiography in patients with RV infarction. ${ }^{14}$ A group of patients developed a rapid restoration of RV function and the reduction of pulmonary pressures.

In the present study, we evaluate the relation between reperfusion as assessed with coronary angiography and the recovery of RV function as assessed by echocardiography. The echocardiographic changes seems to be related to the decrease in pulmonary artery pressure, which suggests that this improvement in RV function is related to the success of reperfusion therapy. The pathophysiology of pulmonary hypertension remains unclear.The hypokinetic right ventricle should not be able to generate hypertension. We suppose that increased pulmonary artery pressure depends on elevated right atrial pressure. In patients with a recovery of RV function, right atrial pressure drops and the pulmonary pressure decreases independently from tri- 
cuspid regurgitation velocity. On the contrary, patients with persistent RV dysfunction had an elevated mean right arterial pressure that influenced the pulmonary pressure. Patients who had persistent RV dysfunction had higher prevalence of right coronary occlusion. This group had higher rates of mortality and complication during the acute phase of myocardial infarction. At baseline, the RV dysfunction was similar in patients who were later successfully or unsuccessfully reperfused, but the clinical outcome was better in patients who were successfully reperfused and promptly recovered right atrial function. Similarly, the LV function was intact in both group of patients, suggesting that the negative outcome depended mostly on RV dysfunction. As compared with the left ventricle, the right ventricle is protected from ischemia because it requires less oxygen because of its small muscular mass and workload. Most of the oxygen is provided by extensive collateral vessels in addition to the direct diffusion of oxygen from the ventricular cavity. 1,21 These factors may limit the size of the RV infarct but do not prevent the hemodynamic failure that results from RV involvement. Reperfusion induced a prompt recovery of ventricular function, a reduction of RV size, an increase in the RV wall motion score index, and a reduction of the tricuspid regurgitant flow, reflecting a reduction in pulmonary pressures.

\section{Limitation of the Study}

Our conclusions are based on the evaluation of coronary angiography performed after thrombolysis. The diagnosis was made with the use of clinical, electrocardiographic, and echocardiographic features. For a better evaluation, angiography should be performed before and after thrombolysis. Bow ers and cow orkers ${ }^{16}$ evaluated reperfusion after percutaneous transluminal coronary angioplasty in their control study.

\section{Conclusions}

In this study, we related the echocardiographic evolution of RV infarction and the angiographic aspects.The persistence of echocardiographic findings of RV dysfunction and abnormal motion of the interventricular and interatrial septum is related to the prognosis of patients with RV infarction. The mortality rate and the incidence of major complications during the in-hospital phase of the study and after discharge were higher in patients with RV dysfunction after thrombolytic therapy who did not experience reperfusion of the related artery.

\section{REFERENCES}

1. Kinch JM, Ryan TJ. Right ventricular infarction. N Engl J M ed 1994;330:1211-7.

2. Braat $S H$, de Z waan $C$, Brugada $P$, Coenegracht J M, Wellens $\mathrm{HJJ}$. Right ventricular involvement with acute inferior wall myocardial infarction identifies high risk of developing atrioventricular nodal conduction disturbances. Am H eart J 1984;107:1183-7.

3. Berger PB, Ryan TJ. Inferior myocardial infarction: high risk subgroups. Circulation 1990;81:401-11.

4. Cohn JN. Right ventricular infarction revisited. Am J Cardiol 1979;43:666-8.

5. Goldstein J A, Barzilai B, R osamond TL, Eisenberg PR, J affe AS. D eterminant of hemodynamic compromise with severe right ventricular infarction. Circulation 1990;82:359-68.

6. Dell'Italia LJ, Starling MR, Crawford MH, Boros BL, Chanduri TK, O'Rourke RA. Right ventricular infarction: identification by hemodynamic measurements before and after volume loading and correlation with noninvasive techniques. J Am Coll Cardiol 1984;4:931-9.

7. I sner J M, Roberts WC. Right ventricular infarction complicating left ventricular infarction secondary to coronary heart disease: frequency, location, associated findings and significance from analysis of 236 necropsy patients with acute or healed myocardial infarction. Am J Cardiol 1978; 42:885-94.

8. Zehender M, Kasper W, Kauder E, Geibel A, Schonthaler M, Olschewski $M$, et al. Eligibility for and benefit of thrombolytic therapy in inferior myocardial infarction: focus on the prognostic importance of right ventricular infarction. J Am Coll Cardiol 1994;24:362-9.

9. Schuler G, H ofmann M, Schwarz F, et al. Effect of successful thrombolytic therapy on right ventricular function in acute inferior wall myocardial infarction. Am J Cardiol 1984;54: 951-7.

10. Steele P, Kirch D, Ellis J, Vogel R, Battock D. Prompt return to normal of depressed right ventricular ejection fraction in acute inferior myocardial infarction. Br H eart J 1977;39: 1319-23.

11. Roth A, Miller H I, Kaluski E, et al. Early thrombolytic therapy does not enhance the recovery of the right ventricle in patients with acute inferior myocardial infarction and predominant right ventricular involvement. Cardiology 1990; 77:40-9.

12. Berger PB, Ruocco NA, Timm CT, Zaret BL, Wackers FJ, and the TIM I Investigators. The impact of thrombolytic therapy in right ventricular infarction complicating inferior myocardial infarction: results from TIMI II. Circulation 1989;80:313-4.

13. Sheehan $F H, B$ raunwald $E, C$ anner $P$, et al. The effect of intravenous thrombolytic therapy on left ventricular function: a report on tissue-type plasminogen activator andstreptochinase from the Thrombolysis in Myocardial Infarction(TIMI Phase I) trial. Circulation 1987;75: 817-29.

14. M attioli AV, Bastia E, M attioli G. D oppler echocardiographic findings in patients with right ventricular infarction. J U Itrasound M ed 1998;17:297-301.

15. Sahn DJ, D eM aria A, Kisslo J, Weiman A. Recommendations regarding quantification in $M$-mode echocardiography: results of a survey of echocardiographic measurements. Circulation 1978;58:1072-83.

16. Bowers TR, O'N eill WW, Grines C, Pica MC, Safian RD, 
Goldstein JA. Effect of reperfusion on biventricular function and survival after right ventricular infarction. $\mathrm{N}$ Engl J Med 1998;338:933-40.

17. M attioli AV. Analysis of interatrial septum using transthoracic echocardiography. Cardiologia 1997;42:257-64.

18. Kircher BJ, H imelman RB, Schiller N B. N on-invasive estimation of right atrial pressure from the inspiratory collapse of the inferior vena cava. Am J Cardiol 1990;66:493-6.

19. Skjaerpe $T, H$ atle $L$. N oninvasive estimation of systolic pres- sure in the right ventricle in patients with tricuspid regurgitation. Eur H eart J 1986;7:704-10.

20. Bates ER, Califf RM, Stack RS, et al. Thrombolysis and Angioplasty in M yocardial Infarction (TAMI-1) trial: influence of infarct location on arterial patency, left ventricular function and mortality. J Am Coll Cardiol 1989;1:12-8.

21. $H$ aupt $H M, H$ utchins $G M, M$ oore $G W$. Right ventricular infarction: role of the moderator band artery in determining infarct size. Circulation 1983;67:1268-72. 